\title{
THE OCCURRENCE OF CANDIDA ALBICANS IN SPUTUM IN DENMARK
}

\author{
BY \\ P. HELMS \\ From Chest Clinics in Jutland
}

(RECEIVED FOR PUBLICATION SEPTEMBER 15, 1955)

It is known that pulmonary moniliasis may complicate treatment with antibiotics (Wolff, 1952; annotations in the Lancet, 1951, 1952; Werner, 1953 ; Browne, 1954 ; Bratlund and Holten, 1954). Recently Bratlund and Holten (1954) have reported cases of moniliasis during treatment with cortisone or corticotrophin.

Viable Candida albicans has not been found outside the living organism (Nilsby and Nordén, 1949). Sharp (1954) has found that the sputum is the most fruitful source for the isolation of Candida albicans. In his series 174 men with pneumonia were examined before treatment, when Candida albicans was isolated from $48 \%$ of spu:a, $26 \%$ of throat swabs, and $3 \%$ of rectal swabs. Probably infection spreads directly from man to man or perhaps the fungus can be transferred from cattle to man with infected milk (Stableforth, 1952), but for the development of moniliasis in relation to treatment with antibiotics or cortisone/corticotrophin the most likely pathogenesis is an activation of an infection present before the onset of treatment. It may therefore be of interest to know how frequently Candida albicans occurs in the sputum of untreated persons.
The sputa were incubated on Sabouraud medium. Cultures of yeasts were transferred to blood-agar medium. The microscopical morphology of the yeasts was examined by the Benham slide method (Benham, 1931). The identification of Candida albicans was made on typical morphology and the presence of characteristic chlamydospores. Table I shows the results of an investigation on the occurrence of Candida albicans in sputum from 205 persons examined during 1952-3 at the chest clinics in the counties of Viborg and Thisted in Jutland. The patients were visiting the clinics for various respiratory diseases and for routine examinations. The diagnoses in the table are based on tuberculin testing, radiological and sputume examinations, the last comprising microscopy and cultivation for tubercle bacilli and for fungi. The examinations of the sputa were undertaken at the Statens Seruminstitut, Copenhagen.

Table I shows that Candida albicans was found in the sputa from 70 to $80 \%$ of the examined persons. The frequency is a little greater in females $(81 \%)$ than in males $(73 \%)$, but is independent of age.

TABLE I

RESULTS OF EXAMINATION OF SPUTUM FOR CANDIDA ALBICANS IN 205 UNTREATED PERSONS

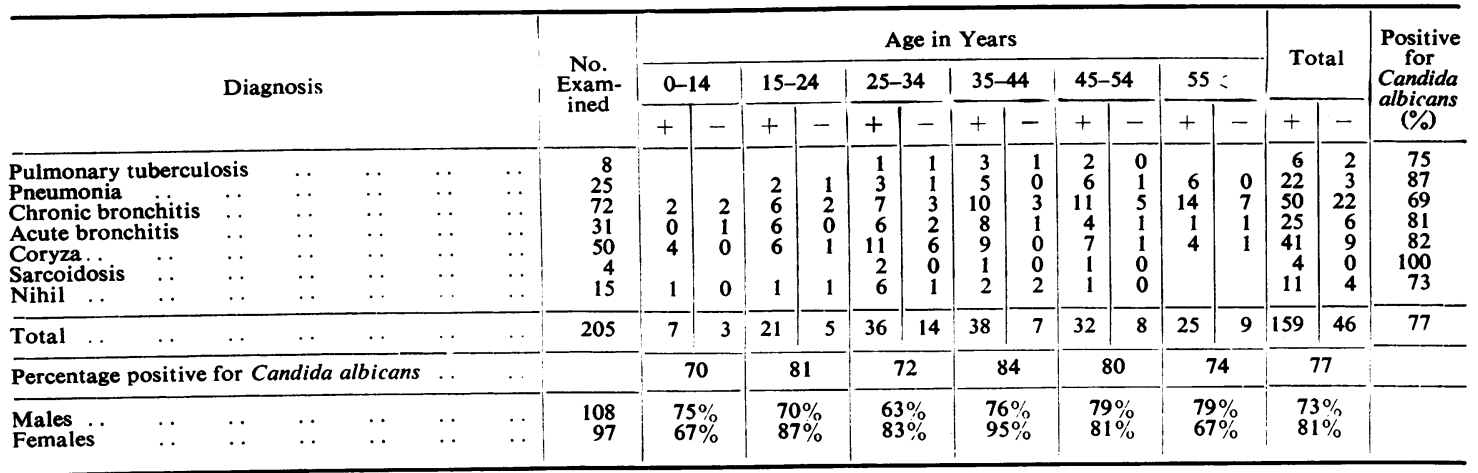


There is no significant variation in the frequency according to diagnosis; Candida albicans was found with the same frequency in persons with the common cold and without demonstrable respiratory disease as in persons with real respiratory disease. It may therefore be concluded that Candida albicans is very frequent in sputum from untreated persons, and three-fourths of those examined may be in danger of developing pulmonary moniliasis. But, in view of the great number of patients with respiratory disease now treated with antibiotics, manifest pulmonary moniliasis seems to be a very uncommon complication. Even when special attention is paid to its possibility it is rarely seen, and case reports have been few. The risk of development of pulmonary moniliasis is therefore not a contraindication to treatment with antibiotics. Only when a febrile pulmonary disease reacts unexpectedly to treatment with antibiotics, especially if the patient deteriorates during continued therapy, must this complication be considered. If the cause of the deterioration is moniliasis the diagnosis is simple ; the sputum will be crowded with yeast-like cells which are easily found microscopically in unstained or simp'y stained smears.

The investigations of Bratlund and Holten (1954) indicate that moniliasis is a much more frequent complication of treatment with cortisone or corticotrophin. They found moniliasis in 8 to $10 \%$ of patients treated with these drugs. It is therefore recommended that patients under longterm treatment with cortisone or corticotrophin should be regularly examined for moniliasis both clinically (thrush) and mycologically.

\section{Summary}

The sputum from 205 untreated persons with and without respiratory disease was examined for Candida albicans. This organism was found in $77 \%$. There was no difference in the frequency according to age and diagnosis.

Pulmonary moniliasis is a rare complication of antibiotic therapy.

According to Bratlund and Holten moniliasis frequently complicates treatment with cortisone/ corticotrophin. It is recommended that patients under treatment with these drugs should be regularly examined for Candida albicans.

I wish to express my thanks to Dr. G. Bindslev. who kindly allowed these examinations to be made on the patients visiting the chest clinics, and to Dr. Per Holm, who made all the mycological examinations and supplied the method.

\section{REFERENCES}

Annotation (1951). Lancet, 2, 532

(1952). Ibid., 1, 1247.

Benham, R. W. (1931). J. infect. Dis., 49, 183.

Bratlund, H., and Holten, C. (1954). Danish med. Bull., 1, 79. Browne, S. G. (1954). Lancet, 1, 393.

Nilsby, I., and Nordèn, A. (1949). Acta med. scand., 133, 340. Sharp, J. L. (1954). Lancet, 1, 390.

Stableforth, A. W. (1952). Ibid., 2, 41.

Werner, G. H. (1953). Ibid., 2, 676.

Wolff, F. W. (1952). Ibid., 1, 1236. 